\title{
Can classical optical superposition principle get us out of quantum mysticism of non-locality and bring back reality to modern physics?
}

\section{Chandrasekhar Roychoudhuri}

Chandrasekhar Roychoudhuri, "Can classical optical superposition principle get us out of quantum mysticism of non-locality and bring back reality to modern physics?," Proc. SPIE 9665, Tenth International Topical Meeting on Education and Training in Optics and Photonics, 966508 (3 June 2007); doi: $10.1117 / 12.2207464$

SPIE Event: Tenth International Topical Meeting on Education and Training in Optics and Photonics, 2007, Ottawa, Ontario, Canada 


\title{
Can classical optical superposition principle get us out of quantum mysticism of non-locality and bring back REALITY to modern physics?
}

\author{
Chandrasekhar Roychoudhuri \\ Photonics Lab., Physics Department, U. Connecticut, Storrs, CT, USA
}

\begin{abstract}
We already know that in the purely technological frontier, photonics is going to play a leading role during this century riding on the shoulders of electronics-, bio- and nano-technologies. This talk will underscore that the fundamentals of photonics as has already been developed by classical optics, specifically, the Superposition Principle (SP) will play a profound role in bringing REALITY in physics while opening up many new paths to innovations by opening up new understandings behind SBP becomes manifest only when the detectors and the detectees are within each others range of interacting forces and can exchange energy allowed by the quantum restrictions to manifest the measurable transformations undergone by them The deeper recognition of this fundamentally "local" SP will guide us overcome many conceptual bottlenecks, appreciate deeper realities hidden behind the current quantum formulation, remove the unnecessary non-causal interpretations of quantum mechanics, bridge classical and quantum optics and bring back conceptual freedoms for many new photonics innovations. One of the many specific examples is that the apparent spectrometric resolution limit $\delta v \delta \mathrm{t}>1$ is not a fundamental principle of nature. I will show mathematical and experimental work to establish this assertion.
\end{abstract}

1. It is generally accepted that photonics will lead the technology innovations of this century. Both science and religion converge on one common opinion that in the beginning of the creation there was light. Today's science continues to reveal the critical functional and facilitating role of light behind almost all the life and evolutionary activities. Without the Sun light there would be no life, period. Down to Earth, in our econo-sphere, the global economic competitive advantage is driven by the rate of innovation toward building intelligent, diverse device-based, multi-functional, micro systems. Micro systems must continuously evolve to higher intelligence through multiple feed-back loops. Inter connectivity required for these feed-back loops between the integrated diverse components will be a critical element to enhance this intelligence. What is going to serve these ubiquitous functions? Light will be the best inter connector and intelligence enhancer. Light can gather and deliver information; it can pick up and deliver energy through and between diverse materials, simply based on the choice of frequency of the radiation and the right lightmatter interaction principle. Science of light is still continuing to make breakthroughs. Nano photonics (manipulating light in domains that are orders of magnitude less than the wavelength) is already developing many new technologies by integrating biological devices and functions. Optics is going to play the role of the critical technology enabler for decades to come riding on the shoulders of all the other matured technologies as their integrators and intelligence enhancer by giving them more"voices of intelligence" through many flexible feed-back loops..

The field of Electronics started only a century ago and it is already almost peaking in technology maturity (rate of patent submission). In contrast, the importance and the role of light as a profoundly important enabler of both science and technology have been recognized by modern human since many centuries past. However, it is as old as the beginning of the universe. The technologies behind galactic evolution has been started by nature with light (The Big Bang)! The Electronic chip owes its existence to very recent understanding of Solid State Physics, empowered by Quantum Mechanics, which itself was forced on us by Spectroscopy (discrete lines of gas discharge) and Black Body radiation. Stimulated emission gave birth to lasers, which is now ubiquitous in our every day life, whether they are technological devices ( $C D$ read-write) or industrial instruments (Raman spectrometers). Spectrometry is the most precise measurement tool ever invented by us. No major field of science or industry can thrive today without these tools. But these fundamental tools could not have been invented without the help of science of light at the fundamental level. We are now waiting to translate Bose-Einstein Condensates (BEC) into new technologies.

2. This century, photonics will also lead the way to bring reality in fundamental physics by giving 'active voice' to the Superposition Principle. Mathematical framework behind the classical superposition principle (SP) was well developed in the process of studying "interference" of light and other sinusoidally undulating phenomena like water and sound waves and various coupled mechanical pendulums and electrical oscillators and transformers. Quantum mechanics rightly co-opted and generalized SP to a higher level. All measurable (observable) transformations in this universe becomes

Tenth International Topical Meeting on Education and Training in Optics and Photonics, edited by Marc Nantel, Proc. of SPIE Vol. 9665, 966508 - (C) 2007 SPIE, OSA, IEEE, ICO doi: $10.1117 / 12.2207464$ 
possible if and only when a detector and a detectee (i) are physically superposed within their range of interaction by an appropriate force and then (ii) the rules of constrained interactions allow the exchange of energy to manifest the sought-after transformation. Thus, SP is universal, but it is an active process. SP cannot become manifest (measurable) without real physical interaction.

2.1. Appreciating that EM fields do not interact with each other inspite of their linear superposition being accepted by Fourier theorem and Maxwell's wave equation. All wave phenomena propagate through each other unperturbed beyond the physical domain of interaction if not perturbed by inserting detectors. Water waves pass through each other; the superposition fringes are visible only in the region of physical superposition and because the medium that manifests the wave phenomenon is directly visible to us. Same is true for sound waves as one can validate by carefully listening to the voice of a friend from a distance in a rowdy party. Same is true for light 'waves'. Otherwise, the visual universe would have been full of space and time scintillating 'interference' patterns. Or, the hair-thin fibers that use WDM technology (wavelength domain multiplexing) would have converted the independent data bits all mixed up; we do know that light does produce beat signal when different frequencies are mixed. But, unlike water waves, the medium that manifests light waves is not directly visible to us. That is why we must insert photo detectors within the physical region of superposition of the light waves. Photo detectors being quantum mechanical (energy levels are quantized and frequency sensitive) and electrons themselves being discrete (quantized), we can only register discrete number of electrons in any photo detection process. This does not unequivocally prove that light constitutes propagating 'bullets' of indivisible energy $h v[1,2,3]$.

That EM fields do not operate on each other is accepted in physics. Yet, somehow we have been overriding this daily observed fact by claiming that there is 'interference' of light even in the absence of detectors as if the superposed fields by themselves can re-distribute their energy in space and/or time. Detectors are atomic size entities. For them to respond to single or superposed multiple beams, they must experience all the fields simultaneously present on themselves before they can absorb energy from the fields and undergo any measurable transformation. Thus any and all measured superposition effects must necessarily be local (within the range EM force of interaction).

It is true that Fourier theorem is mathematically correct in showing the linear superposition relation between multiple sinusoids. We also know that Maxwell's wave equation accepts any single sinusoid or their all possible linear combinations. However, the correctness of mathematical linearity cannot override the real world's necessity of real physical interaction between detector and detectee to generate measurable transformations in the real world. The summation implied by the SP is actually carried out by the detectors while they respond to all the filed amplitudes simultaneously as electric dipoles. Susceptibility (polarizability) takes care of this part of the physics. This is also well known calculation recipe in classical and quantum mechanics. Giving the "active voice" to the SP opens up the door to understand the actual physical processes behind the generation and absence of SP effects based on the quantum restrictions of the detecting dipoles. Coherence theory should be re-written in terms of correlation of dipole stimulations [3].

3. Re-visiting classical spectrometry through "active" Superposition Principle. Is elevating SP as an active interaction process purely semantics? We will summarize the derivation of a generalized theory of spectrometry based on the real physical superposition of light pulses and then show that the classical results are particular case along with better understanding of the physics of (processes behind) spectroscopy. It will also be obvious that spectral super resolution for short pulses can be obtained with precision many orders of magnitudes better than the classical limit set by Fourier's corollary $\delta v \delta t>1$.

Causally speaking, all light signals are pulsed; only the duration may be very short or very long. High resolution spectrometers like gratings and Fabry-Perots (FP) are beam or pulse replicator with a temporal periodicity given by the $\tau=\mathrm{m} \lambda / \mathrm{c}$ where $\mathrm{m}$ is the diffraction order for gratings and the interference order $(2 d / \lambda)$ for FP, $d$ being the plate separation. Then an incident pulse $a(t) \exp [i 2 \pi v t]$ with a carrier frequency $v$ will produce a partially superposed train of pulses [3]. We are presenting the formula for the time varying intensity for a grating only:

$$
\left|i_{\text {out }}(t)\right|^{2}=\left|\sum_{n=0}^{N-1}(1 / N) a(t-n \tau) \cdot \exp [i 2 \pi v(t-n \tau)]\right|^{2}
$$

The corresponding time integrated energy distribution recorded by a photographic plate is:

$$
I_{p l s}(\nu, \tau) \mid=(1 / N)+\left(2 / N^{2}\right) \sum_{p=1}^{N-1}(N-p) \gamma(p \tau) \cos [2 \pi p v \tau]
$$




$$
\begin{aligned}
\text { We have written } \gamma(|m-n| \tau) & \equiv \gamma(p \tau) \text {; this normalized autocorrelation function is defined as: } \\
\gamma(p \tau) & =\int d(t-n \tau) d(t-m \tau) d t / \int d^{2}(t) d t
\end{aligned}
$$

It is interesting to recognize that the total stretch of the pulse train is $\tau_{0}=N \tau$, where $\mathrm{N}$ is the total number of the grating slits. So, whenever the width of the incident pulse a(t) is longer than $\tau_{0}$, the spectral fringe pattern given by the generalized spectrometric Eq. 2 becomes identical to the classical text book formula derived under CW condition:

$$
\underset{\delta t \rightarrow \tau_{0} \equiv N \tau}{L t .} I_{p l s}(v, \tau)=\frac{1}{N}+\frac{2}{N^{2}} \sum_{p=1}^{N-1}(N-p) \cos [2 \pi p v \tau] \equiv \frac{1}{N^{2}} \frac{\sin ^{2} \pi N \nu \tau}{\sin ^{2} \pi \nu \tau} \equiv I_{c w}(v, \tau)
$$

We now have two new physical insights from the Eqns.1-4. Classical spectrometers have a characteristic time constant $\tau_{0}$. When the incident pulse is longer than $\tau_{0}$, we get the classical CW formula. When the pulse width is shorter than $\tau_{0}$, the broadened spectral fringe width is given by Eq.2. But, if we can separately determine $\gamma(\tau)$ through an autocorrelation instrument, we can determine the value of the carrier frequency with arbitrary precision from Eq.2; we are not limited by $\delta v \delta t>1$; it is not a fundamental limit of nature because Fourier theorem is not a principle of nature. It comes from the correct mathematical assumption that numerically the spectral fringe width for a pulse is given by the convolution of the CW fringe pattern of Eq.4 with the mathematical Fourier intensity spectrum $\tilde{A}(v)$ due to the time function a(t). Using Parseval's theorem of conservation of energy, one can mathematically demonstrate that our generalized Eq. 2 can be found equivalent to classical assumption:

$$
I_{p l s}(v, \tau) \approx \int_{-\infty}^{\infty}\left|i_{\text {out }}(t)\right|^{2} d t=I_{c w}(v) \otimes \tilde{A}(v)
$$

We believe that this amthematical equivalency of the convolution of Eq.5 with our generalized formula of Eq. 2 has constrained us in believing that the Fourier corollary $\delta v \delta t>1$ is a limiting principle of nature. The experimental paper that demonstrates super resolution by heterodyne spectroscopy can be found from Ref.3.

4. Future of "active" Superposition Principle in fundamental Physics. Active SP clearly implies that the "dominant part" of the quantum-ness of photo detection process is determined by the quantized photo detectors themselves. That EM fields do not operate on each other is accepted in physics. Yet, somehow we have been overriding this daily observed fact by claiming that there is 'interference' of light even in the absence of detectors as if the superposed fields by themselves can re-distribute their energy in space and/or time. Detectors are atomic size entities. For them to respond to single or multiple superposed beams, they must experience all the fields simultaneously present on themselves before they can absorb energy from the joint-fields and undergo any measurable transformation. Thus any and all measured superposition effects must necessarily be local (within the range EM force of interaction) [4]. It also strengthens us to reject un-supportable non-causal assertions like "non-locality" and "teleportation" in SP experiments. Consider more down to earth observations that contradict accepted physics assumptions. Fourier transform spectroscopy works because Michelson assumed that different optical frequencies do not interfere with each other. His assumption was correct for slow detectors of his time. Today we routinely carry out heterodyne spectroscopy by superposing different frequencies on a fast detector; different frequencies do give SP effects. We still claim that orthogonally polarized light do not interfere with each other. But EM fields do not interfere (interact) with each other, no matter what. The physical reason is that the same detecting molecule, once responded to the stronger EM fields upon them as a dipole, it cannot simultaneously respond as a dipole in the orthogonal direction; so it cannot sum the effect of both the fields and hence there are no fringes when the fields are orthogonally polarized.

5. Acknowledgements. Past support for some of the activities presented here was provided by the Nippon Sheet Glass Corporation.

\section{References}

1. C. Roychoudhuri, Katherine Creath and Al F. Kracklauer, Organizing Editors; "The Nature of Light: What Is a Photon?; Proc. SPIE Vol.5866 (2005); Year of Einstein Special Conference.

2. C. Roychoudhuri \& R. Roy, Organizing Editors, Optics \& Photonics News Trends, The Nature of Light: What is a Photon?"; special issue of OPN, October 2003. [http://www.osaopn.org/abstract.cfm?URI=OPN-14-10-49]

3. Recent publications by the author on this subject: http://www.physics.uconn.edu/ chandra/

4. C. Roychoudhuri, Phys. Essays 19 (3), September 2006; "Locality of superposition principle is dictated by detection processes". 